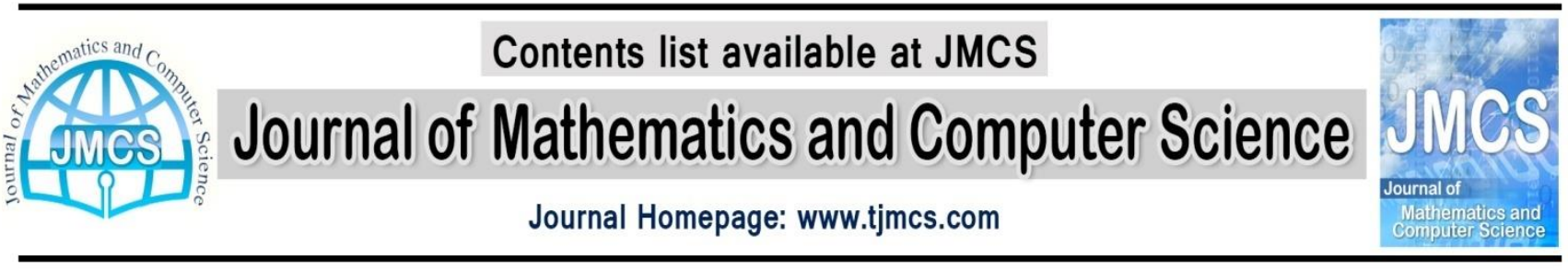

\title{
Equilibrium Problems and Minimax Inequalities for Mappings with Noncompact Domain
}

\author{
Mahdiyeh Farshidzad \\ Department of Mathematics, Sheikh Bahai University, Isfahan, IRAN \\ m.farshidzad@yahoo.com
}

Article history:

Received August 2013

Accepted November 2013

Available online November 2013

\begin{abstract}
In this paper we consider equilibrium problem and introduce minimization problem such as equilibrium problem. We also investigated minimax inequalitiy of Ky Fan and we proved a minimax inequality of mapping with non-compact domain by using the coincidence theorem, then given a minimax inequality for mappings with noncompact domain. As its direct consequences some minimax inequalities and minimax theorems are obtained.
\end{abstract}

Keywords: Equilibrium problem, Minimax theorem, Ky Fan's inequality, Noncompact domain.

\section{Introduction}

Let $X$ be a real topological vector space with topological dual $X^{*}$ denote the duality pairing between $X$ and $X^{*}$ by $\langle\cdot, \cdot\rangle$; Let $K$ be a nonempty subset of $X$.

If $\mathrm{f}$ is a real bifunction defined on $\mathrm{K} \times \mathrm{K}$, the equilibrium problem is denoted:

(EP) find $\bar{y} \in K$ such that $f(x, \bar{y}) \leq 0$, for each $x \in K$

Dual of equilibrium problem is:

(ÉP) find $\bar{x} \in K$ such that $f(\bar{x}, y) \geq 0$, for each $y \in K$.

In this paper we present some examples of equilibrium and minimization problems. By using Fan lemma we study theorems about existence solution of equilibrium problem, then investigate some minimax theorems and minimax inequality. In 1986, by using Browder's selection theorem [2] for the multivalued mapping with open inverse values, Komiya [4] gave the Coincidence theorem. Tarafdar and Watson [7] gave a minimax inequality for mapping with non-compact domain by using the Coincidence theorem. 
Recently Zhang proof a minimax inequality for mappings with noncompact domain .In Sect. 2, we present some examples of equilibrium problem and study theorem about existence solution of equilibrium problem. In Sect. 3 we prove minimax theorem and minimax inequality of KyFan. In Sect. 4 we present Coincidence theorem and with using that prove minimax inequality for non-compact domain. In Sect. 5 we present a new minimax inequality for non-compact domain.

\section{Existence solution of equilibrium problem}

(1) Minimization. Let $\psi: k \rightarrow R$ and consider the minimization problem

.(M) find $\bar{x} \in K$ such that $\forall y \in K \quad \psi(\bar{x}) \leq \psi(y)$.

If we set $\mathrm{f}(\mathrm{x}, \mathrm{y})=\psi(\mathrm{y})-\psi(\overline{\mathrm{x}}) \quad \forall \mathrm{x}, \mathrm{y} \in \mathrm{k} \quad$ then we see that Problems (M) and (EP) ' are equivalent

(2) Saddle Point. Let $F: U \times V \rightarrow R$ be a real bifunction, and consider the saddle-point problem $(\mathrm{SP})$ find $(\overline{\mathrm{u}}, \overline{\mathrm{v}}) \in \mathrm{U} \times \mathrm{V}: \quad \mathrm{F}(\overline{\mathrm{u}}, \mathrm{v}) \leq \mathrm{F}(\overline{\mathrm{u}}, \overline{\mathrm{v}}) \leq \mathrm{F}(\mathrm{u}, \overline{\mathrm{v}}) \quad \forall(\mathrm{u}, \mathrm{v}) \in \mathrm{U} \times \mathrm{V}$.

(3) Vibrational Inequalities. Let $\mathrm{T}: \mathrm{K} \rightarrow \mathrm{X}^{*}$ be an operator, and let us consider the following vibrational inequality problem:

(VIP) find $\overline{\mathrm{x}} \in \mathrm{K}$ such that $\forall \mathrm{y} \in \mathrm{K},\langle\mathrm{T}(\overline{\mathrm{x}}), \mathrm{y}-\overline{\mathrm{x}}\rangle \geq 0$;

Let $\mathrm{f}(\mathrm{x}, \mathrm{y})=\langle\mathrm{T}(\mathrm{x}), \mathrm{y}-\mathrm{x}\rangle$ then we see that problems (VIP) and (EP) ' are equivalent.

For the existence of solutions of equilibrium problems, we shall need the following Fan lemma.

Lemma 2.1. (Fan) Let $X$ be a Hausdorff topological vector space, and let $B$ be a nonempty subset. Consider a set-valued operat $\mathrm{T}: \mathrm{B} \rightarrow 2^{\mathrm{X}}$ such that:

(1) for every $x \in B, T(x)$ is closed and nonempty in $X$;

(2) there exists $x 0 \in B$ such that $T(x,$.$) is compact ;$

(3) for every finite subset $A$ of $B, \operatorname{conv}(A) \subset U_{x \in A} T(x)$;

Then $\cap_{\mathrm{x} \in \mathrm{B}} \mathrm{T}(\mathrm{x}) \neq \varnothing$.

Our first main existence theorem is the following.

Theorem 2.1.(see [2]) Let $X$ be a Hausdorff topological vector space, let $K$ be a nonempty closed convex subset Consider two real bifunctions $\varphi$ and $\psi$ Consider two real bifunctions $\varphi$ and $\psi$ defined on KxK such that:

H1. For each $x, y \in K$, if $\psi(x, y) \leq 0$, then $\varphi(x, y) \leq 0$.

$H 2$. For each fixed $x \in X$, the function $\varphi(x, \cdot)$ is lower semicontinu ous on every compact subset of $K$.

$\sup _{\mathrm{y} \in \operatorname{conv}(\mathrm{A})} \min _{\mathrm{x} \in \mathrm{A}} \Psi(\mathrm{x}, \mathrm{y}) \leq 0$ : H3. For each finite subset $\mathrm{A}$ of $\mathrm{K}$, one has

H4. Compactness Assumption. 
There exists a compact convex sub set C of K such that either (1) or (2) below holds:

(1) for all $y \in K \backslash C$, there exists $x \in C$ such that $\varphi(x, y)>0$;

(2) for all $y \in K \backslash C$ there exists $x_{0} \in C$, such that $\psi\left(x_{0}, y\right)>0$;

Then there exists an equilibrium point $\bar{y} \in C$ (i.e $\varphi(x, \bar{y}) \leq 0$ for each $x \in K$ ).

Furthermore, the set of solutions is compact.

Theorem 2.2. Let $K$ be a convex compact subset of a Hausdorff topological vector space and let, $\mathrm{f}: \mathrm{K} \times \mathrm{K} \rightarrow \mathrm{R}$ be a real bifunction such that:

(1) for each $x \in K, f(x, \cdot)$ is lower semicontinuous;

(2) for each $y \in K, f(\cdot, y)$ is quasiconcave;

Then, there exists $\bar{y} \in K$ such that:

$\sup _{x \in k} f(x, \bar{y}) \leq \sup _{x \in k} f(x, x)$.

Proof. We introduce the bifunctions $\varphi$ and $\psi$ defined on $\mathrm{K}^{\times} \mathrm{K}$ by

$\Phi(\mathrm{x}, \mathrm{y})=\Psi(\mathrm{x}, \mathrm{y})=\mathrm{f}(\mathrm{x}, \mathrm{y})-\sup _{\mathrm{x} \in \mathrm{K}} \mathrm{f}(\mathrm{x}, \mathrm{x})$

Theorem 2.1 implies that there exists $\bar{y} \in K$ such that $\varphi(x, y) \leq 0$ for each $x \in K$.

\section{Minimax Inequalities of Ky Fan}

Definition 3.1. Mapping set-valued $T$ defined on $X$ with values in $Y$ called upper semicontinuous if for each open subset $G$ of $Y$ the set $\{x \in X: T(x) \subset G\}$ is open in $X$.

Lemma 3.1. (See [3]) Let $E$ be a Hausdorff topological vector space and $K \subset E$ be a compact convex subset. Let $Z$ be a $n$-simplex. If $q$ is a upper Semicontinuous set-valued mapping defined on $Z$ such that $q(x)$ is a nonempty closed convex subset of $K$ for each $x \in Z$, and if $P: K \rightarrow Z$ is a (single-valued) valued continous mapping, then there exists $\mathrm{x}_{0} \in \mathrm{Z}$ such that $\mathrm{x}_{0} \in \mathrm{P}\left(\mathrm{q}\left(\mathrm{x}_{0}\right)\right)$.

Theorem 3.2. (See [7]) Let $E$ and $F$ be Hausdorff topological vector spaces, let $X \subset E$ and $Y \subset F$ be nonempty convex subsets, and let $A \subset X \times Y$ be a subset such that:

a. for each $\mathrm{x} \in \mathrm{X}$, the set $\{\mathrm{y} \in \mathrm{Y}:(\mathrm{x}, \mathrm{y}) \notin \mathrm{A}\}$ is convex, or empty;

b. for each $y \in Y$, there exists a closed subset $X_{y} \subset X$ such that $\{x \in X:(x, y) \in A\} \subset X_{y}$;

Suppose that there exists a subset $B$ of $A$ and a compact convex subset $K$ of $X$ such that $B$ is closed in $X \times Y$ and

c. for each $y \in Y$, the set $\{x \in K:(x, y) \in B\}$ is nonempty and convex ;

Then $\bigcap_{\mathrm{y} \in \mathrm{Y}} \mathrm{X}_{\mathrm{y}} \cap \mathrm{K} \neq \varnothing$. 
Theorem 3.3. Let $E$ and $F$ be Hausdorff Hausdorff topological vector spaces and let $X \subset E$ and $Y \subset F$ be nonempty convex subsets. if $\mathrm{f}, \mathrm{g}, \mathrm{h}: \mathrm{X} \times \mathrm{Y} \rightarrow \mathrm{R}$ are function such that:

(1) $\forall(x, y) \in X \times Y \quad f(x, y) \leq g(x, y) \leq h(x, y)$;

(2) $f(x, y)$ is lower semicontinuous on $X$, for each $y \in Y$;

(3) $g(x, y)$ is quasiconcave on $Y$, for each $x \in X$;

(4) $h(x, y)$ is lower semicontinuous on $X x Y$ and $h(x, y)$ is quasiconvex on $X$, for each $y \in Y$;

Then

$$
\inf _{\mathrm{x} \in \mathrm{k}} \sup _{\mathrm{y} \in \mathrm{Y}} \mathrm{f}(\mathrm{x}, \mathrm{y}) \leq \inf _{\mathrm{k} \in \overline{\mathrm{k}}} \sup _{\mathrm{y} \in \mathrm{Y}} \min _{\mathrm{x} \in \mathrm{k}} \mathrm{h}(\mathrm{x}, \mathrm{y})
$$

Where $\overline{\mathrm{K}}=\{\mathrm{K} \subset \mathrm{X} \mid \mathrm{K}$ is convect compact subset of $\mathrm{X}\}$.

In addition, if $X$ compact then $\min _{x \in X} \sup _{y \in Y} f(x, y) \leq \sup _{y \in Y} \min _{x \in X} h(x, y)$.

Proof. We can assume that the right-hand side of (i) is not $+\infty$. Choose a real number $t$ such that $\mathrm{t}>\inf _{\mathrm{k} \in \overline{\mathrm{k}}} \sup _{\mathrm{y} \in \mathrm{Y}} \min _{\mathrm{x} \in \mathrm{K}} \mathrm{h}(\mathrm{x}, \mathrm{y})$.

$B=\{(x, y) \in X \times Y: h(x, y) \leq t\}, A=\{(x, y) \in X \times Y: g(x, y) \leq t\}$. Then Let

(a) $\forall \mathrm{x} \in \mathrm{X}$, by (3), set $\{\mathrm{y} \in \mathrm{Y}:(\mathrm{x}, \mathrm{y}) \notin \mathrm{A}\}$ is convex or empty and satisfies condition (a) of theorem (3.1) ;

(b) for each y $\in Y$, by (1): $\{x \in X:(x, y) \in A\} \subseteq\{x \in X: f(x, y) \leq t\}=X_{Y}$;

By (2) $X_{Y}$ is closed and satisfies condition (b) of theorem (3.1). It is easy to verify that $B$ is closed in $X x Y, B$ $\subset A$, and for any $y \in Y$, set $\{x \in X,(x, y) \in B\}$ is convex.

of $X$ such that: $t>\sup _{y \in Y} \min _{x \in K} h(x, y)$, subset convex Let $K$ be a compact theorem (3.1) Then for any $y \in Y$ the set $\{x \in K: h(x, y) \leq t\}$ is nonempty and convex. Thus by

,$\cap_{\mathrm{y} \in \mathrm{Y}} \mathrm{X}_{\mathrm{y}} \cap \mathrm{K} \neq \varnothing$.

That is there exists $x_{0} \in K$ such that $f\left(x_{0}, y\right) \leq t, \forall y \in Y$ this shows that $\inf _{x \in X} \sup _{y \in Y} f(x, y) \leq t$ and hence, (3.2) is proved.

\section{Corollary .3.1.}

(a) Let $\mathrm{E}$ be a Hausdorff topological vector spaceconvex subsets $\mathrm{Y} \subset \mathrm{E}, \mathrm{X} \subset \mathrm{Y}$ be nonempty convex subset and $\mathrm{X}$ be compact. Let $\mathrm{f}, \mathrm{g}: \mathrm{X} \times \mathrm{Y} \rightarrow \mathrm{R}$ be two real-valued function satisfyin

(1) $\forall(x, y) \in X \times Y \quad, f(x, y) \leq g(x, y)$;

(2) $f(x, y)$ is quasiconvex on $X$, for each $y \in Y$; 
(3) $g(x, y)$ is upper continuous on $Y$, for each $x \in X$;

Then $\inf _{x \in X} f(x, y) \leq \sup _{y \in Y} \inf _{x \in X} g(x, y)$.

(b) Let $E$ be a Hausdorff topological vector space, $X \subset Y, Y \subset E$ be nonempty convex subset and $X$ be compact. Let $\mathrm{f}: \mathrm{X} \times \mathrm{Y} \rightarrow \mathrm{R}$ be a real-valued function such that

(1) $f(x, y)$ is quasiconvex on $X$, for each $y \in Y$;

(2) $f(z, y)$ is upper continuous on $Y$, for each $x \in X$;

Then $\inf _{x \in X} f(x, y) \leq \sup _{y \in Y} \inf _{x \in X} f(x, y)$

\section{Coincidence theorem and related theorems with them}

Definition 4.1. A topological space is said to be contractible if the identity mapping is homotopic to a constant function.

Theorem4.1.(see [4]) Let $X$ be a nonempty convex subset of a linear topological space $E$ and let $Y$ be a nonempty compact convex subset of a linear topological space $F$ Let $A$ be an upper semicontinuous and closed convex-valued mapping of $X$ into $Y$ Let $B$ be a convex-valued mapping of $Y$ into $X$ such that $\mathrm{B}^{-1}(\mathrm{x})$ is open in $\mathrm{Y}$ for each $\mathrm{x}$ in $\mathrm{X}$.

Then there exist $\left(\mathrm{x}_{0}, \mathrm{y}_{0}\right) \in \mathrm{X} \times \mathrm{Y}$ such that $\mathrm{y}_{0} \in \mathrm{A}\left(\mathrm{x}_{0}\right), \mathrm{x}_{0} \in \mathrm{B}\left(\mathrm{y}_{0}\right)$.

Theorem 4.2. (See [6]) Let $\mathrm{X}$ be a contractible space and $\mathrm{Y}$ a Hausdorff compact space. Let $\mathrm{A}: \mathrm{X} \rightarrow 2^{\mathrm{Y}}$ be upper semicontinuous with non-empty compact contractible values. Suppose that $\mathrm{B}: \mathrm{Y} \rightarrow 2^{\mathrm{X}}$ is such that:

a. $\mathrm{B}^{-1}(\mathrm{x}), \forall \mathrm{x} \in \mathrm{X}$ is open;

b. for each open set $S$ in $Y$, the set $\cap_{y \in S} B(y)$ is empty or contractible,

Then there exist $\mathrm{z}_{0} \in \mathrm{A}\left(\mathrm{w}_{0}\right), \mathrm{w}_{0} \in \mathrm{B}\left(\mathrm{z}_{0}\right)$.

Theorem4.3.(see[5]) Let $X$ be a complete convex subset of a locally convex Hausdorff topological vector space $E$, and $Y$ be convex set in Hausdorff topological vector space $H$. Let $g: Y \rightarrow 2^{X}$ upper semicontinuous with nonempty compact convex values and $\mathrm{f}: \mathrm{X} \rightarrow 2^{\mathrm{Y}}$ have nonempty convex values such that:

(1) $\mathrm{f}^{-1}(\mathrm{y}), \forall \mathrm{y} \in \mathrm{Y}$ contains an open set $\mathrm{O}_{\mathrm{y}} \subset \mathrm{X}$;

(2) $\cup_{y \in Y} O_{y}=X$;

(3) There exists a compact convex set $\mathrm{Y}_{0} \subset \mathrm{Y}_{1}, \mathrm{Y}_{1} \subset \mathrm{Y}$ such that $\mathrm{D}=\mathrm{\cap}_{\mathrm{y} \in \mathrm{Y}_{0}} \mathrm{O}_{\mathrm{y}}^{\mathrm{c}}$; is compact or empty (here $\mathrm{O}_{\mathrm{y}}^{\mathrm{c}}$ denotes the complement of $\mathrm{O}_{\mathrm{y}}$ ). 
Then there exists an $\mathrm{x}_{0} \in$ Xsuch that $\mathrm{g}^{-1}\left(\mathrm{x}_{0}\right) \cap \mathrm{f}\left(\mathrm{x}_{0}\right) \neq \varnothing$.

Theorem4.4. Let $\mathrm{X}$ and $\mathrm{Y}$ be as above. Let $\mathrm{f}: \mathrm{Y} \rightarrow 2^{\mathrm{X}}$ be upper semicontinuous with compact convex values and $\mathrm{g}: \mathrm{Y} \times \mathrm{X} \rightarrow \mathrm{R}$ such that:

(1) $\mathrm{x} \mapsto \mathrm{g}(\mathrm{y}, \mathrm{x}), \forall \mathrm{y} \in \mathrm{Y}$ is lower semicontinuous;

(2) $\mathrm{y} \mapsto \mathrm{g}(\mathrm{y}, \mathrm{x}), \forall \mathrm{x} \in \mathrm{X}$ is quasi-concave; and

(3) There exists a nonempty compact convex set $\mathrm{Y}_{0} \subset \mathrm{Y}_{1}, \mathrm{Y}_{1} \subset \mathrm{Y}$ such that for all $\mathrm{z} \in \mathrm{X} \backslash \mathrm{f}\left(\mathrm{Y}_{1}\right)$ there exists a $w \in Y_{0}$ such thatg $(w, z)>\sup _{\substack{y \in Y \\ u \in f(y)}} g(y, u)$. Then

$$
\inf _{\mathrm{x} \in \mathrm{X}} \sup _{\mathrm{y} \in \mathrm{Y}(\mathrm{y})} \mathrm{g}(\mathrm{y}, \mathrm{x}) \leq \sup _{\mathrm{y} \in \mathrm{Y} \text { uff(y) }} \mathrm{g}(\mathrm{y}, \mathrm{u})
$$

Proof. We begin by noting that Condition (3) makes no sense when $\sup _{\substack{\mathrm{y} \in \mathrm{f}(\mathrm{y}) \\ \mathrm{f}}} \mathrm{g}(\mathrm{y}, \mathrm{u})=\infty$ though then the conclusion is trivially satisfied. Without loss of generality, we may assume

$$
\mathrm{r}:=\sup _{\substack{\mathrm{y} \in \mathrm{Y}(\mathrm{y}) \\ \mathrm{u}}} \mathrm{g}(\mathrm{y}, \mathrm{u})<\infty
$$

Suppose, for a contradiction, that $r<\inf _{\mathrm{x} \in \mathrm{X}} \sup _{\mathrm{y} \in \mathrm{Y}} \mathrm{g}(\mathrm{y}, \mathrm{x})$. Then the set-valued mapping $\mathrm{h}: \mathrm{X}$ $\rightarrow 2^{\mathrm{Y}}$ defined by $\mathrm{h}(\mathrm{x})=\{\mathrm{y} \in \mathrm{Y}: \mathrm{g}(\mathrm{y}, \mathrm{x})>r\}$ is nonempty with convex values.

Furthermore $\mathrm{h}^{-1}(\mathrm{y})=\{\mathrm{x} \in \mathrm{X}: \mathrm{g}(\mathrm{y}, \mathrm{x})>r\}=\mathrm{O}_{\mathrm{y}}$ is open as $\mathrm{g}(\mathrm{y},$.$) is lower semicontinuous.$

It is easily seen that $U_{y \in Y} O_{y}=X$. Finally, we show $\cap O_{y \in Y}^{c}$ is compact or empty. For $z \in X \quad f\left(Y_{1}\right)$

employing (3), we have the existence of a $w \in Y_{0}$ such that $g(w, z)>r$.

we get $\left\{\mathrm{z} \in \mathrm{Xf}\left(\mathrm{Y}_{1}\right): \mathrm{g}(\mathrm{w}, \mathrm{z})>r\right\}=\mathrm{O}_{\mathrm{W}}$

that is, there is a $\mathrm{w} \in \mathrm{Y}_{0}$ such that $\mathrm{z} \notin \mathrm{O}_{\mathrm{w}}^{\mathrm{c}}$. This implies $\mathrm{z} \notin \mathrm{O}_{\mathrm{w}}^{\mathrm{c}}$ and so $\mathrm{O}_{\mathrm{y} \in \mathrm{Y}_{0}} \mathrm{O}_{\mathrm{y}}^{\mathrm{c}} \subset \mathrm{f}\left(\mathrm{Y}_{1}\right)$. Theorem (4.3) implies the existence of a $\mathrm{y}_{0} \in \mathrm{Y}$ such $\mathrm{th}$ a $\mathrm{f}\left(\mathrm{y}_{0}\right) \cap \mathrm{h}^{-1}\left(\mathrm{y}_{0}\right) \neq \varnothing$ Let $\mathrm{w}_{0}$ be a member of this intersection. Then $\mathrm{w}_{0} \in \mathrm{h}^{-1}\left(\mathrm{y}_{0}\right)$ so $\mathrm{g}\left(\mathrm{y}_{0}, \mathrm{w}_{0}\right)>r$. contradiction to the definition of $\mathrm{r}$. The proof is complete.

\section{Minimax Inequalities for Mappings with Noncompact Domain}

Theorem 5.1. Let $E, F$ be Hausdorff topological vector spaces, $X \subset E, Y \subset F$ be nonempty convex subsets. Let $\mathrm{f}, \mathrm{g}: \mathrm{X} \times \mathrm{Y} \rightarrow \mathrm{R}$ be two real-valued functions, such that

(1) $\forall(x, y) \in X \times Y \quad, f(x, y) \leq g(x, y) ;$

(2) $f(x, y)$ is quasiconvex on $X$, for each $y \in Y$;

(3) $g(x, y)$ is upper semicontinuous on $Y$, for each $x \in X$; 
If $\mathrm{T}$ is an upper semicontinuous set-valued mapping defined on $\mathrm{X}$, such that

(4) $T x$ is a compact convex subset of $Y$, for each $x \in X$;

(5) for each $x \in X$, there exists a subset $V_{x} \subset Y$, such that $K=U_{x \in X} V_{x} \subset Y, V_{x} \cap T x \neq \varnothing$ is compact convex subset.

Then $\inf _{y \in T x} f(x, y) \leq \sup _{y \in y} \inf _{x \in X} g(x, y)$. (ii)

Proof: First, we can assume that the left-hand side of (ii) is not - $\infty$ choose a real number $t$ such that

$$
\inf _{\mathrm{y} \in \mathrm{Tx}} f(\mathrm{x}, \mathrm{y})>t
$$

Let

$Y_{x}=\{y \in Y: g(x, y) \geq t\}$,

$B=\{(x, y) \in X \times Y: y \in T x\}$,

$A=\{(x, y) \in X \times Y: f(x, y) \geq t\}$,

for each $x \in X$. Then, using (ii), for each $y \in Y$, the set

$\{\mathrm{x} \in \mathrm{X}: \mathrm{f}(\mathrm{x}, \mathrm{y})<t\}=\{\mathrm{x} \in \mathrm{X}:(\mathrm{x}, \mathrm{y}) \notin \mathrm{A}\}$ is convex, or empty as $\mathrm{f}(\mathrm{x}, \mathrm{y})$ is quasiconvex on $\mathrm{X}$ for each $y \in Y$ so $A$ satisfies condition (a) of Theorem (3.1). By Assumptions (1) and (3), for each $x \in X, Y_{X}=$ $\{\mathrm{y} \in \mathrm{Y}: \mathrm{g}(\mathrm{x}, \mathrm{y}) \geq \mathrm{t}\} \supset\{\mathrm{y} \in \mathrm{Y}: \mathrm{f}(\mathrm{x}, \mathrm{y})>t\}=\{\mathrm{y} \in \mathrm{Y}:(\mathrm{x}, \mathrm{y}) \in \mathrm{A}\}, \forall \mathrm{x} \in X$

Condition (b) of Theorem (3.1).

Next, we show that $B$ is closed in $X \times Y$. To see this, let $y_{\alpha} \rightarrow y_{0}, y_{\alpha} \in T\left(x_{\propto}\right), x_{\alpha} \rightarrow x_{0}$, we claimy $\in$ $\mathrm{T}\left(\mathrm{x}_{0}\right)$. Indeed if this is not the case, that is, $\mathrm{y}_{0} \notin \mathrm{T}\left(\mathrm{x}_{0}\right)$. Then $\forall \mathrm{y} \in \mathrm{T}\left(\mathrm{x}_{0}\right)$

there exist an open subset $\mathrm{W}_{\mathrm{y}} \subset \mathrm{Y}$ which contains $\mathrm{y}$ and an open subset $\mathrm{U}_{\mathrm{y}} \subset \mathrm{Y}$ which contains $\mathrm{y}_{0}$ such that $\mathrm{W}_{\mathrm{y}} \cap \mathrm{U}_{\mathrm{y}}=\varnothing$ Note that $\mathrm{T}\left(\mathrm{x}_{0}\right) \subset \mathrm{U}_{\mathrm{y} \in \mathrm{T}\left(\mathrm{x}_{0}\right)} \mathrm{W}_{\mathrm{y}}$ since $\mathrm{T}\left(\mathrm{x}_{0}\right)$ is compact, so there exists finite subset $\left\{\mathrm{y}_{1} \ldots \mathrm{y}_{\mathrm{n}}\right\}$,

such that $\mathrm{T}\left(\mathrm{x}_{0}\right) \subset \mathrm{U}_{\mathrm{i}=1}^{\mathrm{n}} \mathrm{W}_{\mathrm{y}_{\mathrm{i}}}=\mathrm{W}$. Let $\mathrm{U}=\mathrm{\cap}_{\mathrm{i}=1}^{\mathrm{n}} \mathrm{U}_{\mathrm{yi}}$ this implies $\mathrm{y}_{0} \in \mathrm{U}$ and $\mathrm{W} \cap \mathrm{V}=\emptyset$.

As $T$ is an upper semicontinuous, and so there exists an open subset $D$ which contains $x_{0}$, such $t h$ a $t$ for each $\forall \mathrm{x}_{\propto} \in \mathrm{D}, \mathrm{T}\left(\mathrm{x}_{\alpha}\right) \subset \mathrm{W}$. Thus, $\mathrm{T}\left(\mathrm{x}_{\propto}\right) \cap \mathrm{U}=\varnothing$ but this contradicts $\mathrm{y}_{\alpha} \rightarrow \mathrm{y}_{0}$. It follows that $\mathrm{B}$ is closed in $X \times Y$ Finally, using (5), for each $x \in X$, the set $\left.\{y \in K:(x, y) \in B\}=U_{x \in X} V_{x} \cap T_{x}, \forall x \in X\right\}$ convex. So K and B satisfy Condition(c) of Theorem (3.1) thus, by Theorem (3.1), $\cap_{x \in X} Y_{x} \cap K \neq \varnothing$ that is, there exists $\mathrm{y}_{0} \in \mathrm{K}$, such that $\mathrm{g}\left(\mathrm{x}, \mathrm{y}_{0}\right) \geq \mathrm{t}$, for all $\mathrm{x} \in \mathrm{X}$. This show that $\sup _{\mathrm{y} \in \mathrm{Y}} \inf _{\mathrm{x} \in \mathrm{X}} \mathrm{g}(\mathrm{x}, \mathrm{y}) \geq$ $\mathrm{t}$ and hence, (ii)holds .

This completes the proof. 
Theorem 5.2. Let $E$ be a Hausdorff topological vector space, $X \subset K, K \subset Y$, and $Y \subset E$ be nonempty convex subsets and $\mathrm{K}$ be compact. Let $\mathrm{f}, \mathrm{g}: \mathrm{X} \times \mathrm{Y} \rightarrow \mathrm{R}$ be two real-valued functions satisfying Conditions (1)-(3),

$(1) \forall(x, y) \in X \times Y, f(x, y) \leq g(x, y) ;$

(2) $f(x, y)$ is lower semicontinuous on $Y$, for each $x \in X$;

(3) $g(x, y)$ is quasiconcave on $X, \forall y \in Y$;

then $\inf _{\mathrm{y} \in \mathrm{Y}} \sup _{\mathrm{x} \in \mathrm{X}} \mathrm{f}(\mathrm{x}, \mathrm{y}) \leq \sup _{\mathrm{x} \in \mathrm{X}} \mathrm{g}(\mathrm{x}, \mathrm{x})$.

Theorem 5.3. Let $X, Y$, and $K$ be as in Theorem 5.2. Let $f: X \times Y \rightarrow R$ be a real-valued function satisfying Conditions (1)-(2),

(1) $f(x, y)$ is lower semicontinuous on $Y$, for each $x \in X$;

(2) $f(x, y)$ is quasiconcave on $X$, for each $y \in Y$;

Then $\inf _{\mathrm{y} \in \mathrm{Y}} \sup _{\mathrm{x} \in \mathrm{X}} \mathrm{f}(\mathrm{x}, \mathrm{y}) \leq \sup _{\mathrm{x} \in \mathrm{X}} \mathrm{f}(\mathrm{x}, \mathrm{x})$.

\section{References}

[1] F.E. Browder, Coincidence theorems, minimax theorems and variational inequalities, Contemp. Math., vol. 26, Amer Math. Soc., Providence, pp. 67-80, RI (1984).

[2] O. Chadil, Z. chbaniz, and H.Riahi, Equilibium problems with Generalized MonotonBifunctions and Applications to Variational Inequalities, Journal of Optimization Theory and Applications: vol. 105, No. 2, pp. 299 - 323, May (2000).

[3] C.W. Ha, Minimax and fixed point theorems, Math. Ann. 248, 73-77, (1980).

[4] H. Komiya, coincidence theorem and saddle point theorem, proc. Amer. Math. soc. 96, 599-602 (1986).

[5] E. Tarafdar and X.Z. Yuan, A remark on coincidence theorems, proc. Amer .soc. 122 (3), 957 -959, (1994).

[6] E. Tarafdar, and P.J. Watson, Aconcidence point theorm and related results, Appl. Math .let. 11 (1), 37 - 40, (1998).

[7] H.J. Zhang, Minimax Inequalities of Key Fan, Apple math. soc .99 , 980 - 682 (1987 ). 